\title{
Point And Interval Estimation for a Simple Step-Stress Model with Type-II Censoring
}

\author{
N. Balakrishnan ${ }^{1}$, Debasis Kundu ${ }^{2}$, H. K. T. Ng$^{3}$, Nandini Kannan ${ }^{4}$
}

\begin{abstract}
In reliability and life-testing experiments, the researcher is often interested in the effects of extreme or varying stress levels such as temperature, voltage, and load, on the lifetimes of experimental units. Accelerated testing allows the experimenter to increase these stress levels to obtain information on the parameters of the life distributions more quickly than would be possible under normal operating conditions. A special class of accelerated tests are step-stress tests that allow the experimenter to increase the stress levels at fixed times during the experiment. In this article we consider the simple step-stress model under Type-II censoring. We derive the maximum likelihood estimators (MLEs) of the parameters assuming a cumulative exposure model with lifetimes being exponentially distributed. The exact distributions of the MLEs of parameters are obtained through the use of conditional moment generating functions. We also derive confidence intervals for the parameters using these exact distributions, asymptotic distributions and the parametric bootstrap method, and assess their performance through a Monte Carlo simulation study.
\end{abstract}

Keywords and Phrases: Accelerated Testing; step-stress models; cumulative exposure model; maximum likelihood estimation; bootstrap method; conditional moment generating function; Type-II censoring; spacings; exponential distribution.

1 Department of Mathematics and Statistics, McMaster University, Hamilton, Ontario, CANADA. 
${ }^{2}$ Department of Mathematics, Indian Institute of Technology, Kanpur, INDIA.

${ }^{3}$ Department of Statistical Science, Southern Methodist University, Dallas, Texas, USA.

${ }^{4}$ Department of Management Science and Statistics, The University of Texas at San Antonio, 1604 N.West Loop, San Antonio, Texas, USA.

Corresponding Author: N. Balakrishnan 


\section{INTRODUCTION}

In industrial experiments, products that are tested are often extremely reliable with large mean times to failure under normal operating conditions. Consequently, with conventional life-testing experiments under either Type-I or Type-II censoring, it is almost impossible to obtain adequate information about the failure time distribution and its associated parameters. To overcome these problems, the experimenter may resort to accelerated testing wherein the units are subjected to higher stress levels than normal. The accelerated tests may be performed using constant stress or linearly increasing stress levels. Examples include assessing the effects of temperature, voltage, load, vibration etc. on the lifetimes of the product. This accelerated testing helps reduce the time to failure. The data collected from such an accelerated test may then be extrapolated to estimate the underlying distribution of failure times under normal conditions. This process requires a model relating the level of stress and the failure distributions. Some key references in the area of accelerated testing include Nelson and Meeker (1978), Meeker and Hahn (1985), Nelson (1990), Meeker and Escobar (1998), and Bagdonavicius and Nikulin (2002).

The step-stress experiment is a special case of accelerated testing that allows for different conditions at various intermediate stages of the experiment. Here, a random sample of $n$ identical units are placed on a life test under an initial stress level of $x_{0}$. At fixed times $\tau_{1}, \tau_{2}, \ldots, \tau_{m}$, the stress levels are increased to $x_{1}, \ldots, x_{m}$, respectively. If we assume Type-II censoring, the experiment will terminate when a specified number of items $r(\leq n)$ fail. If $r$ is taken to be $n$, then a complete sample would be observed from the step-stress test.

We consider here a simple step-stress model with only two stress levels. This model has been extensively studied in the literature. Miller and Nelson (1983) and Bai, Kim and Lee (1989) determined the optimal time $\tau$ at which to change the stress level from $x_{0}$ to 
$x_{1}$. DeGroot and Goel (1979) developed a Bayesian framework for optimal tests. Khamis and Higgins (1998) considered a cumulative-exposure model similar to the constant-stress Weibull model. Xiong (1998) and Xiong and Milliken (1999) considered inference under the assumption of exponential lifetimes. However, in their work the distributions for the MLEs presented seem to be incorrect, due to the fact that the upper bound $(\tau)$ for the failures that occur at the first stress level does not seem to have been accounted for. More specifically, once we are given that $n_{1} \geq 1$ failures occurred before $\tau$, it is evident that $n t_{1: n}$ can not exceed $n \tau$ and hence can not have an exponential distribution as claimed in Xiong (1998). Moreover, in this case, the order statistics $t_{1: n}, t_{2: n}, \ldots, t_{n_{1}: n}$ are order statistics from right-truncated exponential distribution (as we have stated in Lemma 1) and hence the corresponding spacings are not distributed as exponential as claimed in Xiong (1998). Since these two (erroneous) results were used in Xiong's (1998) derivation, the distributions of the MLEs presented there are erroneous.

Xiong (1998) and Xiong and Milliken (1999) assumed that the mean life of an experimental unit is a log-linear function of the stress level. Based on this assumption, these authors developed inference for the two parameters of the corresponding log-linear link function. Watkins (2001) argued that it is preferable to work with the original exponential parameters, even though the log-linear link provides a simple reparametrization.

In this article, we consider the simple step-stress model under Type-II censoring based on exponential lifetimes. In Section 2, we present the MLEs of the parameters and their exact distributions are derived in Section 3. Using these distributions of the MLEs, we obtain the exact confidence intervals for the unknown parameters in Section 4. We also present the asymptotic distributions of the MLEs and the corresponding asymptotic confidence intervals, as well as confidence intervals based on the parametric bootstrap method. In Section 5, we evaluate the performance of these confidence intervals in terms of probability coverages 
through Monte Carlo simulations. Conclusions are finally drawn in Section 6.

\section{Model Description and MLEs}

Consider the following simple step-stress model: $n$ identical units are placed on a life test and subjected to a stress level $x_{1}$. The experiment continues until a specified number of failures $r$ is observed. Let $\tau$ denote the fixed (known) time at which the stress level is increased. Let $n_{1}$ be the number of failures that occur before $\tau$ and $n_{2}$ the number of failures that occur after $\tau$. If $n_{1}=r$, the test is terminated; otherwise, the experiment continues under the same conditions until time $\tau$. At this time, the stress level is changed to $x_{2}$ and the test continues until a total of $r$ failures is observed. Let $t_{1: n}<t_{2: n}<\ldots<t_{r: n}$ denote the ordered failure times. We further assume that the data come from a cumulative exposure model; see Nelson (1980, 1990). The cumulative exposure model relates the life distribution of the units at one stress level to the life distribution of the units at the next stress level. The model assumes that the residual life of the experimental units depends only on the cumulative exposure the units have experienced, with no memory of how this exposure was accumulated.

The lifetime distributions at stress levels $x_{1}$ and $x_{2}$ are assumed to be exponential with means $\theta_{1}$ and $\theta_{2}$, respectively. Therefore, under the assumptions of the cumulative exposure model, the cumulative distribution function (CDF) $G(t)$ is given by [Xiong (1998)]

$$
G(t)=\left\{\begin{array}{ccc}
F_{1}(t) & \text { if } & 0<t<\tau \\
F_{2}\left(\frac{\theta_{2}}{\theta_{1}} \tau+t-\tau\right) & \text { if } & \tau \leq t<\infty
\end{array},\right.
$$

where

$$
F_{1}(t)=1-e^{-\frac{t}{\theta_{1}}} \text { and } \quad F_{2}(t)=1-e^{-\frac{t}{\theta_{2}}}
$$

The probability density function (PDF) is given by

$$
g(t)=\left\{\begin{array}{ccc}
\frac{1}{\theta_{1}} e^{-\frac{t}{\theta_{1}}} & \text { if } & 0<t<\tau \\
\frac{1}{\theta_{2}} e^{-\frac{t-\tau}{\theta_{2}}-\frac{\tau}{\theta_{1}}} & \text { if } & \tau \leq t<\infty
\end{array},\right.
$$


From (2.3), it follows that the likelihood of the observed data $t_{1: n}<\ldots<t_{r: n}$ is

$$
L\left(\theta_{1}, \theta_{2}\right)=\left\{\begin{array}{lcc}
\frac{c_{1}}{\theta_{1}^{r}} e^{-\frac{1}{\theta_{1}}\left[\sum_{i=1}^{r} t_{i: n}+(n-r) t_{r: n}\right]} & \text { if } & n_{1}=r \\
\frac{c_{1}}{\theta_{2}^{r}} e^{-\frac{1}{\theta_{2}}\left[\sum_{i=1}^{r}\left(\frac{\theta_{2}}{\theta_{1}} \tau+t_{i: n}-\tau\right)+(n-r)\left(\frac{\theta_{2}}{\theta_{1}} \tau+t_{r: n}-\tau\right)\right]} & \text { if } & n_{1}=0 \\
\frac{c_{2}}{\theta_{1}^{n_{1}} \theta_{2}^{n_{2}}} e^{-\frac{1}{\theta_{1}}\left\{\sum_{i=1}^{n_{1}} t_{i: n}+\left(n-n_{1}\right) \tau\right\}-\frac{1}{\theta_{2}}\left\{\sum_{i=n_{1}+1}^{r}\left(t_{i: n}-\tau\right)+(n-r)\left(t_{r: n}-\tau\right)\right\}} & \text { if } & 1 \leq n_{1} \leq r-1,
\end{array}\right.
$$

where $c_{1}=\frac{n !}{r !}$ and $c_{2}=\frac{n !}{n_{1} !\left(r-n_{1}\right) !}$.

From the likelihood function in (2.4), the following observations are clear: If $n_{1}=r$ or $n_{1}=0, \sum_{i=1}^{r} t_{i: n}+(n-r) t_{r: n}$ is a complete sufficient statistic. If $1 \leq n_{1} \leq r-1$, $\left(n_{1}, \sum_{i=1}^{n_{1}} t_{i: n}, \sum_{i=n_{1}+1}^{r} t_{i: n}+(n-r) t_{r: n}\right)$ is a complete sufficient statistic. It is also evident that the MLE of $\theta_{1}$ does not exist if $n_{1}=0$, and the MLE of $\theta_{2}$ does not exist if $n_{1}=r$. The MLEs of $\theta_{1}$ and $\theta_{2}$ exist only when $1 \leq n_{1} \leq r-1$ and may be obtained by maximizing the corresponding likelihood function. The MLEs are given by

$$
\begin{gathered}
\hat{\theta}_{1}=\frac{\sum_{i=1}^{n_{1}} t_{i: n}+\left(n-n_{1}\right) \tau}{n_{1}} \\
\hat{\theta}_{2}=\frac{\sum_{i=n_{1}+1}^{r}\left(t_{i: n}-\tau\right)+(n-r)\left(t_{r: n}-\tau\right)}{r-n_{1}} .
\end{gathered}
$$

The solutions $\hat{\theta}_{1}$ and $\hat{\theta}_{2}$ represent the conditional MLEs of $\theta_{1}$ and $\theta_{2}$, conditional on $1 \leq$ $n_{1} \leq r-1$. As pointed out by Watkins (2001), the estimates of $\theta_{1}$ and $\theta_{2}$ in (2.5) and (2.6) are the same as the estimates given in Xiong (1998). Even though the MLEs in (2.5) and (2.6) are the same as the estimates in Xiong (1998), the confidence intervals derived by Xiong (1998), however, seem to be in error. This can be readily observed by noting that, once we have given that $n_{1} \geq 1$ failures occurred before $\tau, n t_{1: n}$ cannot exceed $n \tau$ and hence cannot have an exponential distribution as claimed in Xiong (1998). Moreover, in this case, the order statistics $t_{1: n} \leq t_{2: n} \leq \cdots \leq t_{n_{1}: n}$ are order statistics from a right-censored exponential distribution (as we have stated in Lemma 1) and, therefore, the corresponding spacings are not exponentially distributed as claimed by Xiong (1998). Since these two are 
used in Xiong's derivation of confidence intervals, the resulting confidence intervals are in error.

It is for this reason that in the following sections we present an exact method for deriving confidence intervals for $\theta_{1}$ and $\theta_{2}$ and also propose an approximate method which is simpler to use in case of large sample sizes.

\section{Conditional Distribution of the MLEs}

Since the derivation of the joint (conditional) PDF of $\hat{\theta}_{1}$ and $\hat{\theta}_{2}$ is extremely complicated, we will instead obtain the marginal (conditional) distributions of the estimators. The derivation will require the inversion of the conditional moment generating function (CMGF). For brevity, let us denote the CMGFs of $\hat{\theta}_{1}$ and $\hat{\theta}_{2}$ given $1 \leq n_{1} \leq r-1$ by $M_{1}(t)$ and $M_{2}(t)$, respectively. Therefore,

$$
\begin{aligned}
& M_{1}(t)=E\left[e^{t \hat{\theta}_{1}} \mid 1 \leq n_{1} \leq r-1\right] \\
& M_{2}(t)=E\left[e^{t \hat{\theta}_{2}} \mid 1 \leq n_{1} \leq r-1\right] .
\end{aligned}
$$

To obtain the distribution of $\hat{\theta}_{1}$, we need to determine the CMGF in (3.1). We may write

$$
M_{1}(t)=\sum_{j=1}^{r-1} E\left[e^{t \hat{\theta}_{1}} \mid n_{1}=j\right] \times P\left[n_{1}=j \mid 1 \leq n_{1} \leq r-1\right] .
$$

Note that the number of failures occurring before $\tau$, viz. $n_{1}$ is a non-negative random variable with probability mass function (PMF) given by

$$
P\left[n_{1}=j\right]=\left(\begin{array}{c}
n \\
j
\end{array}\right)\left(1-e^{-\frac{\tau}{\theta_{1}}}\right)^{j} e^{-\frac{\tau}{\theta_{1}}(n-j)}=p_{j}(\text { say }) \quad \text { for } \quad j=0,1, \ldots, n .
$$

We then have

$$
P\left[n_{1}=j \mid 1 \leq n_{1} \leq r-1\right]=\frac{p_{j}}{\sum_{i=1}^{r-1} p_{i}} .
$$

In order to compute $E\left(e^{t \hat{\theta}_{1}} \mid n_{1}=j\right)$, we need the lemmas presented in the Appendix. 
Using Lemmas 1-3, we can establish the following theorem and the proof of which is presented in Appendix.

Theorem 1. The PDF of $\hat{\theta}_{1}$, conditional on $1 \leq n_{1} \leq r-1$, is given by

$$
f_{\hat{\theta}_{1}}(t)=\sum_{j=1}^{r-1} \sum_{k=0}^{j} c_{j, k} f\left(t-\tau_{j, k} ; j, \frac{j}{\theta_{1}}\right),
$$

where $\tau_{j, k}=\frac{\tau}{j}(n-j+k)$ and

$$
c_{j, k}=\frac{(-1)^{k}}{\sum_{i=1}^{r-1} p_{i}}\left(\begin{array}{l}
n \\
j
\end{array}\right)\left(\begin{array}{l}
j \\
k
\end{array}\right) e^{-\frac{\tau}{\theta_{1}}(n-j+k)} .
$$

To compute $M_{2}(t)$ as defined in (3.2), we need Lemmas 4 and 5 presented in Appendix.

Over second main result, on the conditional PDF of $\hat{\theta}_{2}$, given that $1 \leq n_{1} \leq r-1$, is as follows.

Theorem 2: Conditioned on $1 \leq n_{1} \leq r-1$, the PDF of $\hat{\theta}_{2}$ is given by

$$
f_{\hat{\theta}_{2}}(t)=\sum_{j=1}^{r-1} \frac{p_{r-j}}{\sum_{i=1}^{r-1} p_{i}} f\left(t ; j, \frac{j}{\theta_{2}}\right) .
$$

It is of interest to note that the conditional PDF of $\hat{\theta}_{2}$ is indeed a mixture of gamma densities.

\subsection{Properties of the MLEs}

From the two theorems in the previous subsection, we can derive some simple distributional properties of the MLEs.

Theorem 3. The first two moments of $\hat{\theta}_{1}$ and $\hat{\theta}_{2}$ are

$$
\begin{gathered}
E\left(\hat{\theta}_{1}\right)=\sum_{j=1}^{r-1} \sum_{k=0}^{j} c_{j, k}\left(\tau_{j, k}+\theta_{1}\right), \\
E\left(\hat{\theta}_{1}^{2}\right)=\sum_{j=1}^{r-1} \sum_{k=0}^{j} c_{j, k}\left(\frac{(j+1)}{j} \theta_{1}^{2}+\tau_{j, k}^{2}+2 \theta_{1} \tau_{j, k}\right),
\end{gathered}
$$




$$
\begin{aligned}
& E\left(\hat{\theta}_{2}\right)=\theta_{2} \sum_{j=1}^{r-1} \frac{p_{r-j}}{\sum_{i=1}^{r-1} p_{i}}=\theta_{2}, \\
& E\left(\hat{\theta}_{2}^{2}\right)=\theta_{2}^{2} \sum_{j=1}^{r-1} \frac{p_{r-j}}{\sum_{i=1}^{r-1} p_{i}} \times \frac{(j+1)}{j} .
\end{aligned}
$$

The expressions for the expected values clearly reveal that $\hat{\theta_{1}}$ is a biased estimator of $\theta_{1}$ while $\hat{\theta_{2}}$ is an unbiased estimator of $\theta_{2}$. The expressions for the second moments can be used for finding standard errors of the estimates.

We can also obtain expressions for the tail probabilities by integrating the PDFs in (3.5) and (3.6). These expressions will be used to construct exact confidence intervals later in Section 4.

Theorem 4. The tail probability of $\hat{\theta}_{1}$ is given by

$$
P_{\theta_{1}}\left(\hat{\theta}_{1} \geq b\right)=\sum_{j=1}^{r-1} \sum_{k=0}^{j} c_{j, k} \Gamma\left(j, \frac{j}{\theta_{1}}<b-\tau_{j, k}>\right),
$$

where $\langle x\rangle=\max \{x, 0\}$ and $\Gamma(a, z)=\frac{1}{\Gamma(a)} \int_{z}^{\infty} t^{a-1} e^{-t} d t$ is the incomplete gamma ratio. Similarly, the tail probability of $\hat{\theta}_{2}$ is given by

$$
P_{\theta_{2}}\left[\hat{\theta}_{2} \geq b\right]=\sum_{j=1}^{r-1} \frac{p_{r-j}}{\sum_{i=1}^{r-1} p_{i}} \times \Gamma\left(j, \frac{b j}{\theta_{2}}\right) .
$$

Remark 1. In the model considered above, we do not assume any relationship between the mean failure times under the two stress levels. There may, however, be situations where the mean times to failure are proportional to each other with a known proportionality constant, i.e. $\theta_{2}=\rho \theta_{1}$ for a known $\rho$. In this case, the MLE of $\theta_{1}$ exists for all $n_{1}$, and its' exact distribution may be obtained explicitly.

Remark 2. We could also develop hypothesis tests for $H_{0}: \theta_{2}=\rho \theta_{1}$ for a known $\rho$ using the likelihood ratio method. 


\section{Confidence Intervals}

In this section, we propose different methods of constructing confidence intervals for the unknown parameters $\theta_{1}$ and $\theta_{2}$. The first method is based on the exact conditional distributions of the MLEs derived in Section 3. The second method uses the asymptotic distributions of the estimators to obtain approximate confidence intervals for $\theta_{1}$ and $\theta_{2}$. The last one is based on the parametric bootstrap method.

\subsection{Exact Confidence Intervals}

To construct exact confidence intervals of $\theta_{1}$ and $\theta_{2}$, we need the following assumptions:

$P_{\theta_{1}}\left(\hat{\theta}_{1} \geq b\right)$ and $P_{\theta_{2}}\left(\hat{\theta}_{2} \geq b\right)$ are increasing functions of $\theta_{1}$ and $\theta_{2}$, respectively. This assumption guarantees the invertibility of the pivotal quantities. Several authors including Chen and Bhattacharya (1988), Gupta and Kundu (1998), Kundu and Basu (2000), and Childs et al. (2003) have used this approach for constructing exact confidence intervals in different contexts. The method can be briefly described as follows:

Let $c(\theta)$ be a function such that $P_{\theta_{1}^{\prime}}\left(\hat{\theta}_{1} \geq c\left(\theta_{1}\right)\right)=\frac{\alpha}{2}$. For $\theta_{1}<\theta_{1}^{\prime}$, we have

$$
P_{\theta_{1}^{\prime}}\left(\hat{\theta}_{1} \geq c\left(\theta_{1}^{\prime}\right)\right)=P_{\theta_{1}}\left(\hat{\theta}_{1} \geq c\left(\theta_{1}\right)\right) \leq P_{\theta_{1}^{\prime}}\left(\hat{\theta}_{1} \geq c\left(\theta_{1}\right)\right)=\frac{\alpha}{2}
$$

where the inequality is from the assumption that $P_{\theta_{1}}\left(\hat{\theta}_{1} \geq b\right)$ is an increasing function of $\theta_{1}$. Now, from (4.1) and the assumption stated above, it follows that $c\left(\theta_{1}\right)<c\left(\theta_{1}^{\prime}\right)$, which in turn implies that $c(\theta)$ is an increasing function of $\theta$. Therefore, $c^{-1}(\theta)$ exists uniquely, and is also an increasing function of $\theta$. From (4.1), it follows that

$$
P_{\theta_{1}}\left(c^{-1}\left(\hat{\theta}_{1}\right) \leq \theta_{1}\right)=1-\frac{\alpha}{2}
$$

Therefore, $\theta_{1 L}=c^{-1}\left(\hat{\theta}_{1}\right)$ is the lower bound for the $100(1-\alpha) \%$ confidence interval of $\theta_{1}$. Similarly, we obtain $\theta_{1 U}=d^{-1}\left(\hat{\theta}_{1}\right)$, where $d^{-1}($.$) is the inverse of the function d($.$) obtained$ 
as the solution of the equation

$$
P_{\theta_{1}}\left(\hat{\theta}_{1} \leq d\left(\theta_{1}\right)\right)=\frac{\alpha}{2}
$$

Then $\left(\theta_{1 L}, \theta_{1 U}\right)$ is a $100(1-\alpha) \%$ confidence interval for $\theta_{1}$. Since closed-form expressions for $c($.$) and d($.$) are not available, we need to use iterative technique to compute these limits.$ Note that $\left(\theta_{1 L}, \theta_{1 U}\right)$ can be obtained as the solutions of the following non-linear equations:

$$
\begin{aligned}
\frac{\alpha}{2} & =\sum_{j=1}^{r-1} \sum_{k=0}^{j} \frac{(-1)^{k}}{\sum_{i=1}^{r-1} p_{i}\left(\theta_{1 L}\right)}\left(\begin{array}{l}
n \\
j
\end{array}\right)\left(\begin{array}{l}
j \\
k
\end{array}\right) e^{-\frac{\tau}{\theta_{1 L}}(n-j+k)} \Gamma\left(j, \frac{j}{\theta_{1 L}}<\hat{\theta}_{1}-\tau_{j, k}>\right) \\
1-\frac{\alpha}{2} & =\sum_{j=1}^{r-1} \sum_{k=0}^{j} \frac{(-1)^{k}}{\sum_{i=1}^{r-1} p_{i}\left(\theta_{1 U}\right)}\left(\begin{array}{c}
n \\
j
\end{array}\right)\left(\begin{array}{l}
j \\
k
\end{array}\right) e^{-\frac{\tau}{\theta_{1 U}}(n-j+k)} \Gamma\left(j, \frac{j}{\theta_{1 U}}<\hat{\theta_{1}}-\tau_{j, k}>\right)
\end{aligned}
$$

where, as before

$$
p_{j}\left(\theta_{1}\right)=\left(\begin{array}{c}
n \\
j
\end{array}\right)\left(1-e^{-\frac{\tau}{\theta_{1}}}\right)^{j} e^{-\frac{\tau}{\theta_{1}}(n-j)} .
$$

Using a similar argument, a $100(1-\alpha) \%$ confidence interval for $\theta_{2}$ say $\left(\theta_{2 L}, \theta_{2 U}\right)$ can be obtained as the solutions of the two non-linear equations

$$
\begin{gathered}
\frac{\alpha}{2}=\sum_{j=1}^{r-1} \frac{p_{r-j}}{\sum_{i=1}^{r-1} p_{i}} \Gamma\left(j, \frac{\hat{\theta}_{2} j}{\theta_{2 L}}\right), \\
1-\frac{\alpha}{2}=\sum_{j=1}^{r-1} \frac{p_{r-k}}{\sum_{i=1}^{r-1} p_{i}} \Gamma\left(j, \frac{\hat{\theta}_{2} j}{\theta_{2 U}}\right) .
\end{gathered}
$$

Since the confidence interval for $\theta_{2}$ involves $\theta_{1}$, which is unknown, we replace $\theta_{1}$ by $\hat{\theta}_{1}$ in the expression for $p_{i}$ 's in (4.3) and (4.4).

The procedure outlined above requires that, for fixed $b, P_{\theta_{1}}\left[\hat{\theta}_{1} \geq b\right]$ and $P_{\theta_{2}}\left[\hat{\theta}_{2} \geq b\right]$ be increasing functions of $\theta_{1}$ and $\theta_{2}$, respectively. The complicated structure of these functions makes an analytical proof extremely difficult. Numerical results, however, show that the result is true and a heuristic justification along the line of Chen and Bhattacharya (1988) and Kundu and Basu (2000) may be provided. It is easy, however, to show that for a fixed $\theta_{1}, P_{\theta_{2}}\left[\hat{\theta}_{2} \geq b\right]$ is an increasing function of $\theta_{2}$. 


\subsection{Fisher Information Matrix}

Even though we have presented in the preceding subsection an exact method for constructing confidence intervals for $\theta_{1}$ and $\theta_{2}$, we present in this subsection an approximate method due to its computational ease and also because it provides good probability coverages (close to those of exact confidence intervals) for large sample sizes (as will be shown in Section 5)

In this subsection, we obtain explicit expressions for elements of the Fisher information matrix of $\theta_{1}$ and $\theta_{2}$. We can use the asymptotic normality of MLEs then to construct approximate confidence intervals for $\theta_{1}$ and $\theta_{2}$.

Let $\mathbf{I}\left(\theta_{1}, \theta_{2}\right)=\left(I_{i j}\left(\theta_{1}, \theta_{2}\right)\right), i, j=1,2$, denote the Fisher information matrix of $\theta_{1}$ and $\theta_{2}$, where

$$
I_{i j}\left(\theta_{1}, \theta_{2}\right)=-E\left[\frac{\partial \ln L\left(\theta_{1}, \theta_{2}\right)}{\partial \theta_{i} \partial \theta_{j}}\right] .
$$

We have

$$
\begin{gathered}
I_{11}\left(\theta_{1}, \theta_{2}\right)=E\left[-\frac{n_{1}}{\theta_{1}^{2}}+\frac{2 U_{1}}{\theta_{1}^{3}}\right], \\
I_{12}\left(\theta_{1}, \theta_{2}\right)=0=I_{21}\left(\theta_{1}, \theta_{2}\right), \\
I_{22}\left(\theta_{1}, \theta_{2}\right)=E\left[-\frac{n_{2}}{\theta_{2}^{2}}+\frac{2 U_{2}}{\theta_{2}^{3}}\right],
\end{gathered}
$$

where

$$
\begin{gathered}
U_{1}=\sum_{i=1}^{n_{1}} t_{i: n}+\left(n-n_{1}\right) \tau, \\
U_{2}=\sum_{i=n_{1}+1}^{r}\left(t_{i: n}-\tau\right)+(n-r)\left(t_{r: n}-\tau\right) .
\end{gathered}
$$

From (4.5)-(4.8), the observed Fisher information matrix is given by

$$
\left[\begin{array}{ll}
O_{11} & O_{12} \\
O_{21} & O_{22}
\end{array}\right]=\left[\begin{array}{cc}
n_{1} & 0 \\
\hat{\theta}_{1}^{2} & n_{2} \\
0 & \frac{n_{2}^{2}}{\hat{\theta}_{2}^{2}}
\end{array}\right] .
$$

The variances of $\hat{\theta}_{1}$ and $\hat{\theta}_{2}$ can be obtained through the observed information matrix as

$$
V_{1}=\frac{\hat{\theta}_{1}^{2}}{n_{1}} \text { and } V_{2}=\frac{\hat{\theta}_{2}^{2}}{n_{2}} .
$$


The asymptotic distributions of the pivotal quantities $\frac{\hat{\theta}_{1}-E\left(\theta_{1}\right)}{\sqrt{V_{1}}}$ and $\frac{\hat{\theta}_{2}-E\left(\theta_{2}\right)}{\sqrt{V_{2}}}$ may then be used to construct $100(1-\alpha) \%$ confidence intervals for $\theta_{1}$ and $\theta_{2}$ respectively. The $100(1-\alpha) \%$ confidence interval for $\theta_{1}$ and $\theta_{2}$ are then given by

$$
\left[\hat{\theta}_{1}-\left(\sum_{j=1}^{r-1} \sum_{k=0}^{j} c_{j, k} \tau_{j, k}\right) \pm z_{1-\frac{\alpha}{2}} \sqrt{V_{1}}\right]
$$

and

$$
\left[\hat{\theta}_{2} \pm z_{1-\frac{\alpha}{2}} \sqrt{V_{2}}\right]
$$

respectively, where $z_{q}$ is the $q$-th upper percentile of a standard normal distribution.

\subsection{Bootstrap Confidence Intervals}

In this subsection, we construct confidence intervals based on the parametric bootstrap, using the bias-corrected and accelerated (BCA) percentile bootstrap method (see Efron and Tibshirani (1982, pp. 184-188)). To obtain the BCA percentile bootstrap confidence intervals for $\theta_{1}$ and $\theta_{2}$, we use the following algorithm:

\section{Parametric bootstrap:}

1. Based on the original sample $\mathbf{t}=\left(t_{1: n}, t_{2: n}, \ldots, t_{r: n}\right)$, obtain $\hat{\theta}_{1}$ and $\hat{\theta}_{2}$, the MLEs of $\theta_{1}$ and $\theta_{2}$, respectively.

2. Simulate the first $r$ order statistics from a sample of size $n$ from uniform $(0,1)$ distribution, $U_{1: n}, U_{2: n}, \ldots, U_{r: n}$.

3. Find $n_{1}$ such that $U_{n_{1}: n} \leq 1-\exp \left(-\frac{\tau}{\hat{\theta}_{1}}\right)<U_{n_{1}+1: n}$

4. For $j \leq n_{1}, T_{j: n}=-\hat{\theta}_{1} \ln \left(1-U_{j: n}\right)$. For $j=n_{1}+1, \ldots, r, T_{j: n}=-\hat{\theta}_{2} \ln \left(1-U_{j: n}\right)+$ $\tau-\frac{\hat{\theta}_{2}}{\hat{\theta}_{1}} \tau$ 
5. Compute the MLEs of $\theta_{1}$ and $\theta_{2}$ based on $T_{1: n}, T_{2: n}, \ldots, T_{n_{1}: n}, T_{n_{1}+1: n}, T_{n_{1}+2: n}, \ldots, T_{r: n}$, say $\hat{\theta}_{1}^{(1)}$ and $\hat{\theta}_{2}^{(1)}$.

6. Repeat Steps $2-5 B$ times and obtain $\hat{\theta}_{1}^{(1)}, \hat{\theta}_{2}^{(1)}, \hat{\theta}_{1}^{(2)}, \hat{\theta}_{2}^{(2)}, \ldots, \hat{\theta}_{1}^{(B)}, \hat{\theta}_{2}^{(B)}$.

7. Arrange $\hat{\theta}_{1}^{(1)}, \hat{\theta}_{1}^{(2)}, \ldots, \hat{\theta}_{1}^{(B)}$ in ascending order and obtain $\hat{\theta}_{1}^{[1]}, \hat{\theta}_{1}^{[2]}, \ldots, \hat{\theta}_{1}^{[B]}$. Similarly, arrange $\hat{\theta}_{2}^{(1)}, \hat{\theta}_{2}^{(2)}, \ldots, \hat{\theta}_{2}^{(B)}$ in ascending order and obtain $\hat{\theta}_{2}^{[1]}, \hat{\theta}_{2}^{[2]}, \ldots, \hat{\theta}_{2}^{[B]}$.

A two-sided $100(1-\alpha) \%$ BCA bootstrap confidence interval of $\theta_{i}$, say $\left[\theta_{i L}^{*}, \theta_{i U}^{*}\right]$, is then given by

$$
\theta_{i L}^{*}=\hat{\theta}_{i}^{\left(\left[B \alpha_{1 i}\right]\right)}, \quad \theta_{i U}^{*}=\hat{\theta}_{i}^{\left(\left[B\left(1-\alpha_{2 i}\right)\right]\right)}, i=1,2 .
$$

where

$$
\alpha_{1 i}=\Phi\left(\hat{z}_{0 i}+\frac{\hat{z}_{0 i}+z_{\frac{\alpha}{2}}}{1-\hat{a}_{i}\left(\hat{z}_{0 i}+z_{\frac{\alpha}{2}}\right)}\right) \quad \text { and } \quad \alpha_{2 i}=\Phi\left(\hat{z}_{0 i}+\frac{\hat{z}_{0 i}+z_{1-\frac{\alpha}{2}}}{1-\hat{a}_{i}\left(\hat{z}_{0 i}+z_{1-\frac{\alpha}{2}}\right)}\right) .
$$

Here, $\Phi(\cdot)$ is the standard normal cumulative distribution function and the value of biascorrection $\hat{z}_{0 i}$ can be computed as

$$
\hat{z}_{0 i}=\Phi^{-1}\left(\frac{\text { number of } \hat{\theta}_{i}^{(j)}<\hat{\theta}_{i}}{B}\right), \quad i=1,2,
$$

where $\Phi^{-1}(\cdot)$ denotes the inverse function of standard normal cumulative distribution function; the acceleration $\hat{a}_{i}$ is

$$
\hat{a}_{i}=\frac{\sum_{j=1}^{r}\left(\hat{\theta}_{i(\cdot)}-\hat{\theta}_{i(j)}\right)^{3}}{6\left[\sum_{j=1}^{r}\left(\hat{\theta}_{i(\cdot)}-\hat{\theta}_{i(j)}\right)^{2}\right]^{3 / 2}}, \quad i=1,2,
$$

where $\hat{\theta}_{i(j)}$ is the MLE of $\theta_{i}$ based on the original sample with the $j$-th observation $t_{j: n}$ deleted (i.e. the jackknife estimate), $j=1,2, \ldots, r$ and $\hat{\theta}_{i(\cdot)}=\sum_{j=1}^{r} \hat{\theta}_{i(j)} / r$.

We evaluate the performance of all these confidence intervals using a simulation study and an illustrative example in Section 5. 


\section{Simulation Study}

To evaluate the performance of all the methods described in Section 4, we conducted a simulation study. The results for different $n, r$ and $\tau$ are presented in Tables 1 and 2 . The results are based on an average over 1000 replications.

From the tables, we observe that while the exact confidence interval has its probability coverage to be quite close to the pre-fixed nominal level, the BCA bootstrap method has its probability coverage to be quite close to the fixed nominal level for $\theta_{2}$ (see Tables 1 and 2) but the probability coverage for $\theta_{1}$ is not satisfactory (see Table 1 ) though it improves slightly for large $n$ (see Table 2), and the approximate method has its probability coverage to be quite different from the nominal level. Moreover, the probability coverages of the approximate methods are most often smaller than the nominal level. This indicates that the confidence intervals obtained by this method will most often be unduly narrower (see the results in Table 4, for example).

When $\tau$ is small, there will be fewer failures before $\tau$ and so inference for $\theta_{1}$ will not be very precise. As $\tau$ increases, the coverage probability for $\theta_{1}$ gets closer to the nominal value, however the coverage probability for $\theta_{2}$ moves away from the nominal value. This is to be expected, since large values of $\tau$ would provide more information about $\theta_{1}$ as compared to $\theta_{2}$. It is important to observe the performance of the approximate method. For all the nominal levels considered, the coverage probabilities are much lower for small values of $\tau$. A possible reason for this may be due to the fact that the exact distribution of the MLEs is highly skewed and hence, to use the asymptotic normality of the MLEs we require a much larger sample size. This is the reason why even for $n=35$ and $r=28$ in Table 2 , the approximate method does not provide close results.

Based on this simulation study, we recommend the use of the exact method for any 
Table 1: Estimated coverage probabilities (in \%) based on 1000 simulations with $\theta_{1}=12.0$ and $\theta_{2}=4.5, n=20, r=16, B=1000$.

\begin{tabular}{|c|rrr|rrr|rrr|}
\hline C.I. of $\theta_{1}$ & \multicolumn{3}{|c|}{$90 \%$ C.I. } & \multicolumn{3}{c|}{ 95\% C.I. } & \multicolumn{3}{c|}{$99 \%$ C.I. } \\
\hline$\tau$ & Boot & Approx & Exact & Boot & Approx & Exact & Boot & Approx & Exact \\
\hline 1 & 97.7 & 74.0 & 93.6 & 99.0 & 74.7 & 95.8 & 99.9 & 91.7 & 98.9 \\
2 & 98.5 & 83.6 & 88.0 & 99.6 & 84.3 & 94.6 & 100.0 & 93.2 & 99.1 \\
3 & 85.4 & 83.5 & 89.0 & 85.7 & 86.6 & 94.0 & 96.8 & 92.0 & 98.8 \\
4 & 84.2 & 81.8 & 88.4 & 92.1 & 87.0 & 94.2 & 94.5 & 93.7 & 98.5 \\
5 & 93.8 & 85.4 & 91.4 & 96.8 & 89.0 & 95.8 & 97.8 & 93.6 & 98.9 \\
6 & 95.3 & 87.1 & 90.7 & 97.3 & 89.8 & 95.8 & 99.0 & 95.3 & 99.0 \\
\hline C.I. of $\theta_{2}$ & \multicolumn{3}{|c|}{$90 \%$ C.I. } & & \multicolumn{3}{c|}{$95 \%$ C.I. } & & $99 \%$ C.I. \\
\hline$\tau$ & Boot & Approx & Exact & Boot & Approx & Exact & Boot & Approx & Exact \\
\hline 1 & 90.7 & 88.7 & 90.9 & 94.8 & 92.8 & 95.8 & 97.4 & 96.2 & 99.5 \\
2 & 90.1 & 86.1 & 90.5 & 94.3 & 91.1 & 95.8 & 98.1 & 95.0 & 99.5 \\
3 & 89.8 & 87.1 & 91.9 & 94.2 & 91.0 & 96.1 & 97.6 & 95.4 & 99.7 \\
4 & 89.4 & 86.2 & 90.5 & 94.5 & 90.2 & 96.1 & 97.7 & 94.5 & 99.8 \\
5 & 89.7 & 86.6 & 91.0 & 93.8 & 89.9 & 96.0 & 97.8 & 93.7 & 100.0 \\
6 & 88.3 & 84.7 & 91.0 & 93.4 & 87.4 & 96.2 & 97.4 & 92.5 & 99.9 \\
\hline
\end{tabular}

sample size as it provides probability coverages quite close to the pre-fixed nominal levels for both $\theta_{1}$ and $\theta_{2}$. The use of the parametric BCA bootstrap method can be supported for $\theta_{2}$ for all sample sizes, but for $\theta_{1}$ only for large sample sizes since the probability coverages are not satisfactory in this case for small sample sizes. The approximate method can be used when $r$ and $n$ are large due to its computational ease as well as for having its probability coverages close to the pre-fixed nominal level, but this method should not be used when $r$ and $n$ are not large. Finally, it should be mentioned here that we did attempt to use the nonparametric bootstrap method, and found the corresponding coverage probabilities to be extremely poor compared to nominal levels, and hence is not suitable for use in this context.

\subsection{Numerical Example}

In this subsection, we consider the simulated data set presented earlier by Xiong (1998). The parameters chosen by Xiong are $\theta_{1}=e^{2.5}=12.18, \theta_{2}=e^{1.5}=4.48$ and $\tau=5$ with $n=20$ 
Table 2: Estimated coverage probabilities (in \%) based on 1000 simulations with $\theta_{1}=12.0$ and $\theta_{2}=4.5, n=35, r=28, B=1000$.

\begin{tabular}{|c|rrr|rrr|rrr|}
\hline C.I. of $\theta_{1}$ & \multicolumn{3}{|c|}{ 90\% C.I. } & \multicolumn{3}{c|}{ 95\% C.I. } & \multicolumn{3}{c|}{ 99\% C.I. } \\
\hline$\tau$ & Boot & Approx & Exact & Boot & Approx & Exact & Boot & Approx & Exact \\
\hline 1 & 97.0 & 86.4 & 95.0 & 98.4 & 86.6 & 98.8 & 99.6 & 93.3 & 100.0 \\
2 & 81.1 & 84.4 & 90.4 & 91.6 & 88.2 & 93.6 & 96.0 & 93.7 & 99.1 \\
3 & 89.4 & 88.7 & 89.2 & 93.0 & 91.1 & 94.5 & 98.4 & 95.7 & 98.7 \\
4 & 95.8 & 86.9 & 90.7 & 97.9 & 90.2 & 95.1 & 99.2 & 95.4 & 99.1 \\
5 & 94.1 & 89.6 & 89.4 & 97.1 & 92.5 & 94.8 & 99.4 & 95.8 & 98.7 \\
6 & 94.0 & 88.3 & 90.3 & 96.7 & 91.7 & 95.9 & 98.2 & 96.2 & 98.7 \\
\hline C.I. of $\theta_{2}$ & \multicolumn{3}{|c|}{$90 \%$ C.I. } & & \multicolumn{3}{c|}{$95 \%$ C.I. } & & $99 \%$ C.I. \\
\hline$\tau$ & Boot & Approx & Exact & Boot & Approx & Exact & Boot & Approx & Exact \\
\hline 1 & 90.1 & 88.8 & 89.9 & 94.4 & 93.1 & 94.3 & 96.2 & 96.6 & 98.6 \\
2 & 88.5 & 87.0 & 88.4 & 93.5 & 92.4 & 94.0 & 98.4 & 96.4 & 99.1 \\
3 & 89.2 & 87.7 & 88.9 & 94.4 & 92.3 & 94.6 & 98.3 & 96.3 & 99.4 \\
4 & 89.6 & 85.6 & 90.1 & 95.1 & 89.4 & 94.2 & 98.6 & 94.9 & 99.8 \\
5 & 89.4 & 88.4 & 90.3 & 94.0 & 91.8 & 95.7 & 98.1 & 95.6 & 99.6 \\
6 & 89.3 & 85.8 & 91.8 & 93.9 & 90.0 & 96.5 & 97.1 & 94.8 & 99.6 \\
\hline
\end{tabular}

Table 3: Simulated Sample presented by Xiong (1998)

\begin{tabular}{|c||c|c|c|c|c|c|}
\hline \multicolumn{1}{|c||}{ Stress level } & \multicolumn{6}{|c|}{ Failure Time } \\
\hline$\theta_{1}=e^{2.5}$ & 2.01 & 3.60 & 4.12 & 4.34 & \\
\hline$\theta_{2}=e^{1.5}$ & 5.04 & 5.94 & 6.68 & 7.09 & 7.17 & 7.49 \\
\cline { 2 - 7 } & 7.60 & 8.23 & 8.24 & 8.25 & 8.69 & 12.05 \\
\hline
\end{tabular}

and $r=16$. The data is presented in Table 3 .

In this example, we have $n_{1}=4$ and $n_{2}=r-n_{1}=12$. To illustrate the estimation procedure here, the MLEs of $\theta_{1}$ and $\theta_{2}$ are found from (2.5) and (2.6) to be $\hat{\theta}_{1}=23.5175$ and $\hat{\theta}_{2}=5.0558$, respectively. The estimates match the values obtained by Xiong (1998) using the log-linear link. We also provide confidence intervals for the parameters using the three methods in Table 4. From the results, it is seen that the exact confidence intervals are wider in general than the other two intervals. It is also seen that the approximate method always provide narrower confidence intervals while the BCA bootstrap method provides sometimes narrower and at other times wider confidence intervals. This is so because the coverage 
Table 4: Interval Estimation for the Simulated Sample presented by Xiong (1998)

\begin{tabular}{|c||c|c|c|}
\hline \multicolumn{1}{|c||}{} & \multicolumn{3}{c|}{ C.I. for $\theta_{1}$} \\
\hline & $90 \%$ & $95 \%$ & $99 \%$ \\
\hline Bootstrap C.I. & $(7.78,34.05)$ & $(6.03,34.05)$ & $(4.03,34.05)$ \\
Approx C.I & $(0.00,35.66)$ & $(0.00,39.36)$ & $(0.00,46.60)$ \\
Exact C.I. & $(11.70,72.95)$ & $(10.35,94.78)$ & $(8.26,168.97)$ \\
\hline & \multicolumn{3}{c|}{ C.I. for $\theta_{2}$} \\
\hline \multicolumn{1}{|c||}{} & $90 \%$ & $95 \%$ & $99 \%$ \\
\hline Bootstrap C.I. & $(5.76,11.43)$ & $(5.51,12.80)$ & $(4.90,13.72)$ \\
Approx C.I & $(2.66,7.46)$ & $(2.20,7.92)$ & $(1.27,8.82)$ \\
Exact C.I. & $(3.33,8.80)$ & $(3.07,9.86)$ & $(2.64,12.53)$ \\
\hline
\end{tabular}

probabilities for the approximate method are significantly lower than the nominal levels while those of the bootstrap method are sometimes lower and at other times larger than the nominal levels (see Tables 1 and 2).

We also note from Table 4 that the confidence intervals for $\theta_{2}$ are considerably narrower than those for $\theta_{1}$. The primary reason for this is that when $\tau$ is small relative to $\theta_{1}$ (as in the present example), we expect relatively small (large) numbers of failures to occur before (after) $\tau$, thus resulting in high (low) variability in the estimation of $\theta_{1}\left(\theta_{2}\right)$.

Finally, it is important to mention that all the confidence intervals presented in Table 4 are unconditional in the sense that they are based on $n_{1}$ being a random variable taking values between 1 and $r-1$. While conditional confidence intervals can instead be constructed based on the given data with the realized value of $n_{1}$ (rather than treating it as random) which may indeed be narrower, yet, inference drawn from such intervals will be restricted only to the given data, and not applicable for any future experiments. 


\section{Conclusions}

In this article, we have considered the simple step-stress model when the observed data comes from an exponential distribution under Type-II censoring. We have obtained the MLEs of the exponential parameters and obtained their exact distributions. Several different procedures for constructing confidence intervals have been suggested. Results of a simulation study are presented comparing the performance of the different procedures. A numerical example has been provided to illustrate the methods outlined in this article.

From our simulation study, we recommend the use of the exact method in the case of small sample sizes ( $n$ up to 50) since the other two methods have unsatisfactory probability coverages in this case (bootstrap being not satisfactory for $\theta_{1}$ and approximate method being not satisfactory for both $\theta_{1}$ and $\theta_{2}$ ). For larger sample sizes, we recommend the approximate method because of its computational ease and also for having its probability coverages close to the pre-fixed level.

\section{A Lemmas and Proof of Theorems}

Lemma 1: Let $X_{1: n}<\ldots<X_{n: n}$ be the order statistics of a random sample of size $n$ from a continuous distribution with $\operatorname{PDF} f(x)$. Let $D$ denote the number of order statistics less than or equal to some pre-fixed number $\tau$. The conditional joint PDF of $X_{1: n}, \ldots, X_{j: n}$, given that $D=j$, is identical to the joint PDF of all order statistics of size $j$ from the right truncated density function

$$
f_{\tau}(t)= \begin{cases}\frac{f(t)}{F(\tau)} ; & \text { for } 0<t<\tau \\ 0 & \text { otherwise }\end{cases}
$$

where $F($.$) is the distribution function of f($.$) .$

Proof of Lemma 1: See Arnold, Balakrishnan, and Nagaraja (1992, pp. 23-24). 
Lemma 2: Let $Z$ be a continuous random variable with PDF

$$
f_{Z}(z)=\left\{\begin{array}{l}
\frac{\frac{1}{\theta_{1}} e^{-\frac{z}{\theta_{1}}}}{1-e^{-\frac{\tau}{\theta_{1}}}}, \quad \text { if } 0<z<\tau . \\
0 \quad \text { otherwise. }
\end{array}\right.
$$

Then the MGF of $Z$ is

$$
M_{Z}(t)=E\left(e^{t Z}\right)=\frac{1-e^{-\frac{\tau}{\theta_{1}}\left(1-\theta_{1} t\right)}}{\left(1-e^{-\frac{\tau}{\theta_{1}}}\right)\left(1-\theta_{1} t\right)} .
$$

Proof of Lemma 2: The proof is straightforward.

Lemma 3: Let $Y$ be a $\operatorname{Gamma}(\alpha, \lambda)$, i.e. a gamma random variable with shape parameter $\alpha$ and scale parameter $\lambda$, respectively. The PDF of $Y$ is given by

$$
f(y ; \alpha, \lambda)= \begin{cases}\frac{\lambda^{\alpha}}{\Gamma(\alpha)} y^{\alpha-1} e^{-\lambda y} & \text { for } y>0 \\ 0 & \text { otherwise. }\end{cases}
$$

For any arbitrary constant $A$, the MGF of $Y+A$ is

$$
M_{Y+A}(t)=e^{t A}\left(1-\frac{t}{\lambda}\right)^{-\alpha}
$$

Proof of Lemma 3: The proof follows readily from the definition of the moment generating function.

Lemma 4: Let $T_{1: n}<\ldots<T_{r: n}$ be the first $r$ order statistics from the density function as defined in (2.3). The conditional joint PDF of $T_{n_{1}+1: n}, \ldots, T_{r: n}$, given that $n_{1}=j$, where $1 \leq j \leq r-1$ is given by

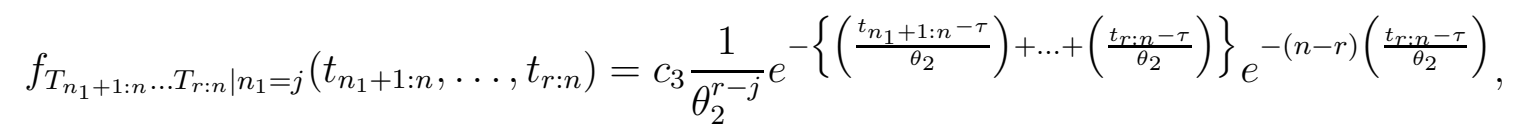

for $\tau<t_{n_{1}+1: n}<\ldots<t_{r: n}<\infty$, where $c_{3}$ is the normalizing constant.

Proof. Using Lemma 1, it is clear that the conditional joint PDF of $T_{n_{1}+1: n}<\ldots<T_{r: n}$, given that $0<t_{1: n}<\ldots<t_{n_{1}: n}<\tau$, is

$$
c_{4} \frac{1}{\theta_{2}^{k}} e^{-\left(\frac{t_{n_{1}+1: n-\tau}}{\theta_{2}}\right)} \ldots e^{-\left(\frac{t_{r \cdot n}-\tau}{\theta_{2}}\right)} e^{-(n-r)\left(\frac{t_{r \cdot n}-\tau}{\theta_{2}}\right)}
$$


for $\tau<t_{n_{1}+1: n}<\ldots<t_{r: n}<\infty$, where $c_{4}$ is the normalizing constant.

\section{Lemma 5:}

$$
M_{2}(t)=\sum_{j=1}^{r-1} \frac{p_{r-j}}{\sum_{i=1}^{r-1} p_{i}}\left(1-\frac{t \theta_{2}}{j}\right)^{-j},
$$

where $p_{j}$ 's are as defined in (3.3).

Proof of Lemma 5 From Lemma 4, and the results on the distribution of spacings of an exponential distribution [see Arnold, Balakrishnan, and Nagaraja (1992, pp. 72-73)], it follows that

$$
E\left(e^{\frac{t \hat{\theta}_{2}}{\theta_{2}}} \mid n_{1}=j\right)=\left(1-\frac{t}{r-j}\right)^{-(r-j)}
$$

which leads to (A.4).

\section{Proof of Theorem 1:}

Using Lemma 1 and Lemma 2 and from (2.5) it easily follows that

$$
E\left(e^{t \hat{\theta}_{1}} \mid n_{1}=j\right)=\frac{e^{\frac{t}{j}(n-j) \tau}\left(1-e^{-\frac{\tau}{\theta_{1}}\left(1-\frac{\theta_{1} t}{j}\right)}\right)^{j}}{\left(1-e^{-\frac{\tau}{\theta_{1}}}\right)^{j}\left(1-\frac{\theta_{1} t}{j}\right)^{j}} .
$$

Therefore,

$$
\begin{aligned}
E\left(e^{t \hat{\theta}_{1}} \mid 1 \leq n_{1} \leq r-1\right) & =\frac{1}{\sum_{i=1}^{r-1} p_{i}} \sum_{j=1}^{r-1}\left(\begin{array}{l}
n \\
j
\end{array}\right) \frac{e^{-\frac{\tau}{\theta_{1}}(n-j)} e^{\frac{t}{j}(n-j) \tau}\left(1-e^{-\frac{\tau}{\theta_{1}}\left(1-\frac{\theta_{1} t}{j}\right)}\right)^{j}}{\left(1-\frac{\theta_{1} t}{j}\right)^{j}} \\
& =\frac{1}{\sum_{i=1}^{r-1} p_{i}} \sum_{j=1}^{r-1} \sum_{k=0}^{j}(-1)^{k}\left(\begin{array}{c}
n \\
j
\end{array}\right)\left(\begin{array}{l}
j \\
k
\end{array}\right) \frac{e^{\frac{t \tau}{j}(n-j+k)} e^{-\frac{\tau}{\theta_{1}}(n-j+k)}}{\left(1-\frac{\theta_{1} t}{j}\right)^{j}} \\
& =\sum_{j=1}^{r-1} \sum_{k=0}^{j} c_{j, k} e^{\frac{t \tau}{j}(n-j+k)}\left(1-\frac{\theta_{1} t}{j}\right)^{-j} \cdot
\end{aligned}
$$

From (A.6), using the inversion theorem of a moment generating function, we compute the conditional PDF of $\hat{\theta}_{1}$ given $1 \leq n_{1} \leq r-1$. We denote the above conditional PDF as 
$f_{\hat{\theta}_{1}}(t)$ for brevity. Comparing (A.6) with Lemma 3, we can easily see that the conditional PDF has the form in (3.5).

\section{$\underline{\text { References }}$}

Arnold, B.C., Balakrishnan, N. and Nagaraja, H.N. (1992), A First Course in Order Statistics, John Wiley \& Sons, New York.

Bagdonavicius, V. and Nikulin, M. (2002), Accelerated Life Models : Modeling and Statistical Analysis, Chapman \& Hall/CRC Press, Boca Raton, Florida.

Bai, D.S., Kim, M.S. and Lee, S.H. (1989), "Optimum simple step-stress accelerated life test with censoring", IEEE Transactions on Reliability, vol. 38, 528-532.

Chen, S.M. and Bhattacharya, G.K. (1988), "Exact confidence bound for an exponential parameter under hybrid censoring", Communications in Statistics - Theory and Methods, vol. 16, 1857-1870.

Childs, A., Chandrasekar, B., Balakrishnan, N. and Kundu, D. (2003), "Exact likelihood inference based on Type-I and Type-II hybrid censored samples from the exponential distribution", Annals of the Institute of Statistical Mathematics, vol. 55, 319-330.

DeGroot, M.H. and Goel, P.K. (1979), "Bayesian estimation and optimal design in partially accelerated life testing", Naval Research Logistics Quarterly, vol. 26, 223-235.

Efron, B. and Tibshirani, R. (1993), An Introduction to the Bootstrap, Chapman \& Hall, New York.

Gupta, R.D. and Kundu, D. (1998), "Hybrid censoring schemes with exponential failure distribution", Communications in Statistics- Theory and Methods, vol. 27, 3065-3083.

Khamis, I. H. and Higgins, J. J. (1998), "A new model for step-stress testing", IEEE Transactions on Reliability, vol. 47, 131-134. 
Kundu, D. and Basu, S. (2000), "Analysis of incomplete data in presence of competing risks", Journal of Statistical Planning and Inference, vol. 87, 221-239.

Meeker, W. Q. and Escobar, L. A. (1998), Statistical Method for Reliability Data, John Wiley \& Sons, New York.

Meeker, W. Q. and Hahn, G. J. (1985), How to Plan Accelerated Life Tests: Some Practical Guidelines, vol. 10 of the ASQC Basic References in Quality Control: Statistical Techniques, available from the American Society for Quality Control, Milwaukee, Wisconsin.

Miller, R. and Nelson, W.B. (1983), "Optimum simple step-stress plans for accelerated life testing", IEEE Transactions on Reliability, vol. 32, 59-65.

Nelson, W. B. and Meeker, W. Q. (1978), "Theory for optimum accelerated censored life tests for Weibull and extreme value distributions" Technometrics, vol. 20, 171-177.

Nelson, W.B. (1980), "Accelerated life testing: Step-stress models and data analysis", IEEE Transactions on Reliability, vol. 29, 103-108.

Nelson, W.B. (1990), Accelerated Life Testing, Statistical Models, Test Plans, and Data Analysis, John Wiley \& Sons, New York.

Watkins, A.J. (2001), "Commentary: inference in simple step-stress models", IEEE Transactions on Reliability, vol. 50, 36-37.

Xiong, C. (1998), "Inferences on a simple step-stress model with Type-II censored exponential data", IEEE Transactions on Reliability, vol. 47, 142-146.

Xiong, C. and Milliken, G. A. (1999), "Step-stress life-testing with random stress-change times for exponential data", IEEE Transactions on Reliability, vol. 48, 141-148. 